\title{
Surface screening of written ferroelectric domains in ambient conditions
}

\author{
J. J. Segura, ${ }^{1,2}$ N. Domingo, ${ }^{1}$ J. Fraxedas,${ }^{1}$ and A. Verdaguer ${ }^{1, a)}$ \\ ${ }^{1}$ Centre d'Investigació en Nanociència i Nanotecnologia, CIN2 (CSIC-ICN), Esfera UAB, Campus de la UAB, \\ Edifici CM-7, 08193-Bellaterra, Catalunya, Spain \\ ${ }^{2}$ Department of Materials Science and Engineering, Ecole Polytechnique Fédérale de Lausanne, \\ 1015 Lausanne, Switzerland
}

(Received 1 October 2012; accepted 12 February 2013; published online 8 May 2013)

\begin{abstract}
We have combined Piezoresponse Force Microscopy and Kelvin Probe Force Microscopy (KPFM) to study screening charge dynamics in written domains on $\mathrm{PbZr}_{0.2} \mathrm{Ti}_{0.8} \mathrm{O}_{3}(\mathrm{PZT})$ thin film surfaces under a controlled environment and at variable temperature. The screening dynamics decayed exponentially on a timescale of tens of minutes, consistently with what we expected for water-mediated surface diffusion of ionic species. Variable-temperature KPFM measurements showed variations in surface potential due to temporary unbalanced surface screening charges. Low humidity experiments revealed gradual incorporation of positive charges onto the surface, even in a non-reactive environment $\left(\mathrm{N}_{2}\right)$, as well as deceleration of the screening dynamics upon reversal of the temperature variation. Our work may serve as a guide for future studies on the dynamics and nature of adsorbates on polarized PZT thin films. (C) 2013 AIP Publishing LLC [http://dx.doi.org/10.1063/1.4801983]
\end{abstract}

\section{INTRODUCTION}

Water layers are present on all surfaces exposed to ambient conditions. They directly affect tribology and wear, surface conductivity, chemical reactivity, and other mechanical and electrical properties. ${ }^{1}$ Given the nanoscale dimensions (angstroms to several nanometers) of these water layers, Scanning Probe Microscopy (SPM) has become a natural choice for studying their properties. ${ }^{2}$ Water layers are also crucial to the mobility of other adsorbates on the surface; these adsorbates and their movement often determine the mechanisms of charge diffusion and surface screening. Surface screening is a general feature of oxide surfaces in air and it strongly affects SPM measurements of the electrostatic and transport properties of surfaces, which can lead to incorrect data interpretation. ${ }^{3}$ Studying the dynamics and nature of the adsorbates involved in the screening is of paramount importance in ferroelectric materials. ${ }^{4}$ Surface charge screening determines the stabilization of the ferroelectric domains $^{5}$ as well as the motion of wall domains. ${ }^{6}$ Electrostatic Atomic Force Microscopy (AFM) techniques such as Kelvin Probe Force Microscopy (KPFM) have been used to study ferroelectric domains and surface screening on $\mathrm{BaTiO}_{3}$ (100) surfaces. ${ }^{7-9}$ In this case, the surface potential at room temperature exhibits variations due to the differences in charge density between different polarized domains. In those studies, it was established that the surface potential takes the sign of the screening charges and therefore, is opposite to what would be expected from the polarization orientation (in the KPFM images, positive domains appear as negative and negative domains appear as positive). Above the critical temperature $\left(T_{c}\right)$, ferroelectric polarization disappears. However, the researchers observed that the morphology of the potential features remained stable and that

\footnotetext{
a) Author to whom correspondence should be addressed. Electronic mail: Albert.Verdaguer.ICN@uab.cat.
}

potential differences between different domains even increased. These potential differences above $T_{c}$ were found to decay temporally, on the order of tens of minutes. These results can be explained on the basis that the new potential distribution (i.e., that reached after the temperature is increased past $\mathrm{T}_{\mathrm{c}}$ ) induces the presence of uncompensated surface charges as the ferroelectric polarization disappears. The unstable potential distribution relaxes with time to a new equilibrium surface potential. Similar effects can be observed when the polarization of the sample changes after the temperature is decreased below $\mathrm{T}_{\mathrm{c}}$. However, in the aforementioned experiments, the researchers did not apply any specific environmental control and, therefore, they did not consider the effects induced by the presence of water. In independent experiments on $\mathrm{BaTiO}_{3}$ run under controlled relative humidity $(\mathrm{RH})$, the surface potential was found to depend on both the electric field and the humidity. ${ }^{10,11}$ This dependence was related to the adsorption and desorption of the surface screening charges, mainly induced by water.

In the work we report here, we sought to study the influence of water on the surface screening processes on perovskite PZT (here, $\mathrm{PbZr}_{0.2} \mathrm{Ti}_{0.8} \mathrm{O}_{3}$ ) thin films grown on Nb-doped $\mathrm{SrTiO}_{3}$ (STO) as a model surface for ferroelectric materials. PZT has a critical temperature $\left(\mathrm{T}_{\mathrm{c}}\right)$ of $490^{\circ} \mathrm{C}$. In the ferroelectric phase, a dipole is generated by the displacement of the Ti ions against the octahedral oxygen atoms. The thin films used in this study were oriented in such that the displacement of the $\mathrm{Ti}$ atoms would be perpendicular to the surface, thereby proving a positive surface dipole when the Ti atoms are displaced upwards, and a negative one if they are displaced downwards. When the film is grown, natural domains of both up and down polarizations are formed spontaneously. The domains depend on many factors, such as the substrate used and the film thickness. Local polarization (writing or poling) of the ferroelectric film can be performed using an AFM tip by applying a bias between the tip and the sample. ${ }^{12}$ In fact, AFM lithography can be used to generate 
well-defined polarized regions that are stable over time. The domains can be characterized by Piezoresponse Force Microscopy (PFM) - namely, by exploiting the piezoelectric response of the system to an AC voltage. ${ }^{13}$ Surface dipoles and charge screening can be studied using KPFM. In KPFM, the contact potential difference (CPD) between the tip and the substrate is measured. Quantitative data on surface potential are very difficult to obtain in KPFM, unless the surface potential of the tip is well characterized. This problem can be partially avoided if the objective of the study is to measure the difference in CPD among distinct regions of an image, which is known as surface-potential contrast $(\Delta \phi)$. One drawback of writing domains with an AFM in ferroelectric thin films is that it implies injection of charges into the surface. Although some work has been done to minimize charge injection, ${ }^{14,15}$ this effect cannot be easily avoided, and the injected charges, which are principal determinant of KPFM contrast, usually overscreen the polarization bond charge. However, injected charges diffuse on the surface, and equilibrium between the new polarization bond charge and the surface charges can be reached on a timescale of tens of minutes. Once in equilibrium, the surface charge dynamics can be studied by varying the sample temperature because polarization-bond charge depends on temperature. ${ }^{8}$ Control of the environment is critical in such studies. By reducing the $\mathrm{RH}$, surface charge dynamics can be slowed down ${ }^{16}$ and the incorporation of new adsorbates can be minimized, thereby facilitating a better understanding of the processes involved.

\section{EXPERIMENTAL DETAILS}

PZT samples $\left(100 \mathrm{~nm}\right.$ thick $\mathrm{PbZr}_{0.2} \mathrm{Ti}_{0.8} \mathrm{O}_{3}$ grown on $\mathrm{Nb}$-doped STO) were acquired from PHASIS (Geneva, Switzerland). The samples were rinsed with ethanol and MilliQ water several times and dried with $\mathrm{N}_{2}$ before experiments.

Experiments were carried out with an MFP-3D Asylum Research Atomic Force Microscope (Asylum Research, Santa Barbara, California, USA). In all the AFM modes used in this work (contact AFM [c-AFM], amplitude modulation AFM [AM-AFM], KPFM, and PFM), the experiments were performed using DPER14 Pt-coated silicon tips ( $\mu$ Masch) with a nominal resonant frequency of $160 \mathrm{kHz}$ and a force constant of $6 \mathrm{~N} / \mathrm{m}$. Large areas $(20 \mu \mathrm{m} \times 20 \mu \mathrm{m}$ or $50 \mu \mathrm{m} \times 50 \mu \mathrm{m})$ were scanned several times in c-AFM in order to remove the maximum amount of contamination from the sample. However, even after the surface conditioning, the sample still showed some nanometer-sized spots associated with contamination. Topography and piezoresponse images of a $4 \mu \mathrm{m} \times 4 \mu \mathrm{m}$ area of the sample, inside the largest area (which was cleaned), were then obtained using a new tip in the Dual AC Resonance Tracking PFM (DARTPFM $)^{17}$ mode [Fig. 1(a)]. Images were acquired at a constant

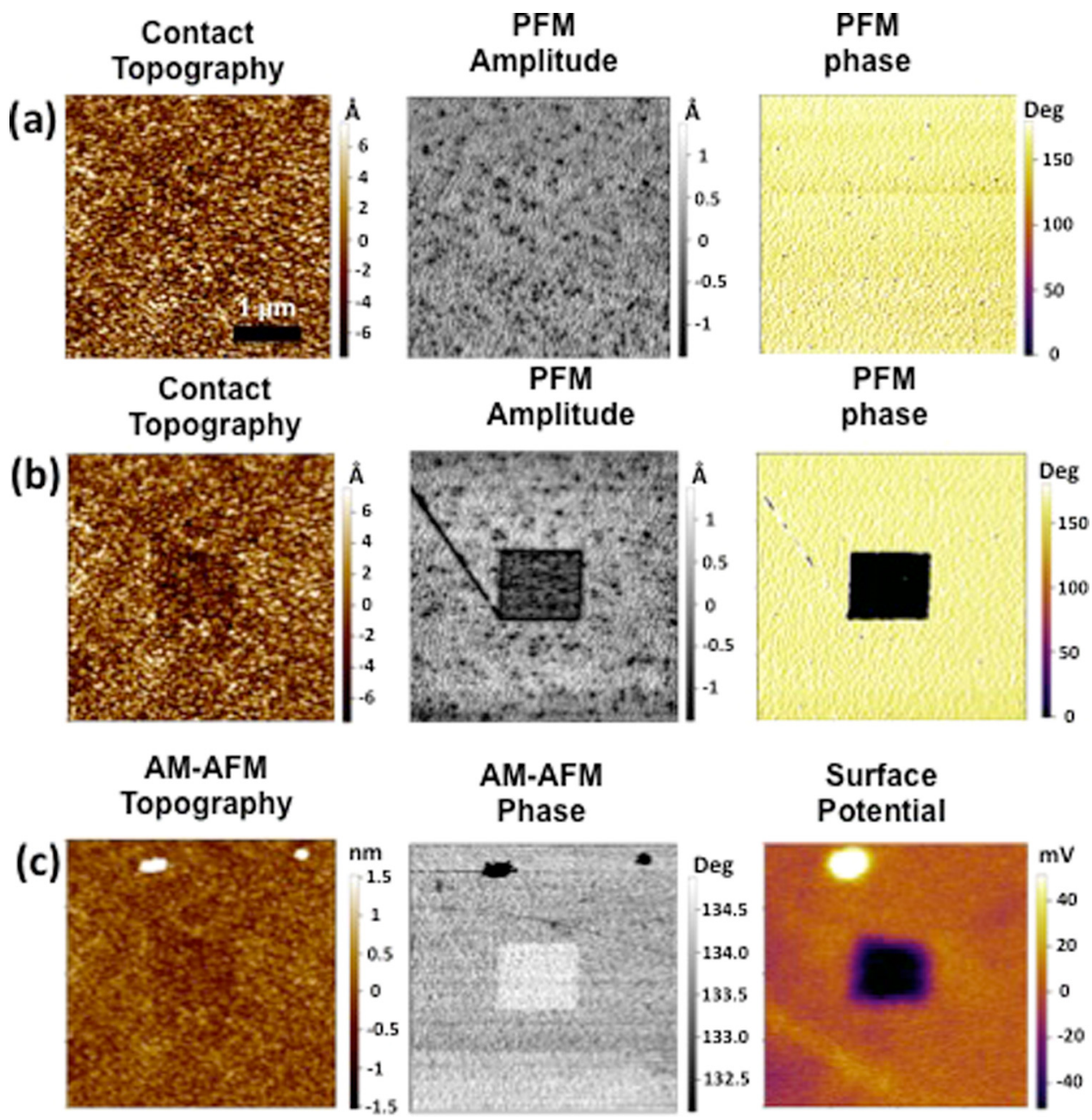

FIG. 1. (a) Contact and PFM AFM images of a $100 \mathrm{~nm}$-thick PZT film grown on a Nb-doped STO substrate. (b) Contact and PFM AFM images of the same region after scanning a $1 \mu \mathrm{m} \times 1 \mu \mathrm{m}$ square in the center of the image while applying a voltage of $+6 \mathrm{~V}$ to the tip. Inversion of polarization of the square is detected in the PFM images as a $180^{\circ}$ phase contrast relative to the nonpolarized region, indicating an opposite polarized direction. (c) AM-AFM and KPFM images of the same region. The contrast of the polarized region is dark relative to that of the non-polarized region, indicating that the effect of the created dipoles had been measured and that it had not been completely screened by surface charges. 
load of $\sim 750 \mathrm{nN}$ and applying an AC voltage (amplitude: $500 \mathrm{mV}$ ) to the tip. After imaging the working area, polarization of the sample was performed in c-AFM by scanning a $1 \mu \mathrm{m} \times 1 \mu \mathrm{m}$ square in the center of the working area while applying a $6 \mathrm{~V}$ bias between the AFM tip and the back of the sample with a $750 \mathrm{nN}$ load. No AC voltage was applied during the polarization procedure. Immediately after polarization, a new DART-PFM image of the whole $4 \mu \mathrm{m} \times 4 \mu \mathrm{m}$ working area was taken to verify the polarization of the sample [Fig. 1(b)]. Once the polarization was verified, topography AM-AFM images were obtained using the same probe [Fig. 1(c)]. CPD images were simultaneously obtained with AM-AFM, using the KPFM mode operated in the lift mode. The minimum time needed between the end of the polarization process and the end of the KPFM image was $\sim 10 \mathrm{~min}$, so that the time origin (zero) of the experiment was set at the end of the first KPFM image acquired.

We measured the CPD contrast between the polarized and non-polarized areas of the sample as a function of $\mathrm{RH}$ and of temperature in a controlled environment chamber. $\mathrm{RH}$ was controlled by circulating dry $\mathrm{N}_{2}\left(\mathrm{H}_{2} \mathrm{O}\right.$ concentration: $<2 \mathrm{ppm}$ ) to decrease RH or by bubbling $\mathrm{N}_{2}$ through Milli-Q water to increase RH. The RH was measured using a Digitron hygrometer. The temperature of the sample was controlled using a Peltier cooling system with nominal precision $\left( \pm 1^{\circ} \mathrm{C}\right)$ and temperature range from $-20^{\circ} \mathrm{C}$ to $50^{\circ} \mathrm{C}$. To minimize the perturbation of the water layers adsorbed on the surface and surface diffusion of adsorbates, the AMAFM was operated in a true, non-contact attractive regime. ${ }^{18}$

\section{RESULTS}

Figure 1 shows c-AFM (topography) and PFM (amplitude and phase) images before (Fig. 1(a)) and after (Fig. 1(b)) polarization of the $1 \times 1 \mu \mathrm{m}$ square. The topography roughness was measured from the contact image, and a value of $\sim 3 \AA$ was obtained. In the PFM amplitude and phase images before polarization, small domains of the film can be observed, although at this resolution level, the sample appears to exhibit a single monodomain orientation. In the PFM amplitude image after polarization, a well-defined $1 \mu \mathrm{m} \times 1 \mu \mathrm{m}$ square can be observed, which shows $180^{\circ}$ phase contrast, indicating that the domain formed was polarized opposite to the main orientation of the sample in its initial (as-received) state. The polarized region was found to be stable in the timeframe of one experiment (i.e., 4 to $8 \mathrm{~h}$ ). Humidity was critical to the polarization process, since it was very difficult to obtain a good polarization when working at low levels of humidity. Thus, all the polarizations were done at ambient humidity (RH $\sim 40 \%$ ). Since a positive voltage $(+6 \mathrm{~V})$ was applied during polarization, negative dipoles were expected to form on the ferroelectric film surface (due to the downwards displacement of the Ti ions far from the positive biased tip). However, at the same time, positive charges were injected during polarization, and an excess of injected positive charges induced a positive surface-potential contrast. The KPFM contrast observed after polarization varied greatly in function of the experimentfrom positive to negative values, and in some cases, even negligible contrast-suggesting that the degree of charge injection was different in each experiment. Charge injection depends on many factors that were beyond experimental control, such as the quality of the tip used or the residual contamination on the sample.

After the above experiments were completed, polarized regions showing a negative or almost null contrast from the first KPFM image were studied (see Fig. 1(c)). The charge injection in those regions was assumed to be lower, and the main contribution to the potential contrast was the presence of the new dipoles, which had not yet been totally screened by surface charges.

We studied the time evolution of the contrast in the contact-potential image under ambient conditions $\left(25^{\circ} \mathrm{C}\right.$ and $40 \% \mathrm{RH})$. A representative example is shown in Figure 2(a): a sample that had shown a negative CPD contrast $(\Delta \phi=-58 \mathrm{mV})$ immediately after polarization was left to relax for $1 \mathrm{~h}$ at $40 \% \mathrm{RH}$; it contrast decayed to $\Delta \phi \sim-3 \mathrm{mV}$. The CPD contrast decay was fitted to an exponential function showing a time constant of $15 \mathrm{~min}$. Since polarization remains stable on this timescale, the decay of the CPD contrast must have been due to surface screening processes.

Surface screening due to the diffusion of ionic species on the surface has been studied for charged nanostructures on non-conductive samples such as graphene sheets deposited on $\mathrm{SiO}_{2}{ }^{19}$ Injected charge in graphene sheets using an AFM tip has been studied by KPFM, which revealed that CPD contrast of the charged graphene decayed exponentially. The time constant of the exponential decay was found to depend on RH: values of $50 \mathrm{~min}$ for $10 \% \mathrm{RH}$ and $10 \mathrm{~min}$ for $50 \% \mathrm{RH}$ were observed. The discharge process was associated to the diffusion of ionic species on the surface due to the presence of adsorbed water layers.

Our results shown in Fig. 2(a) are very similar to those reported for the charged graphene sheets mentioned above, suggesting that the main discharge mechanism is caused by a surface diffusion process due to the presence of water. Other discharge mechanisms in the process must be considered, such as band-bending or reorientation of water dipoles, but these are generally expected to be much faster and to occur before the first KPFM image is taken.

To study the change in CPD contrast as a function of the temperature, we reduced the $\mathrm{RH}$ of the chamber to nearly $0 \%$ (by introducing dry $\mathrm{N}_{2}$ ). Although some water molecules probably remained on the surface even at this very low level of humidity, we expected a drastic reduction in the ionic species mobility on the surface. ${ }^{16}$ The RH in the vicinity of the sample-and consequently, the amount of water that is adsorbed on the sample - depends on the quantity of ambient water as well the temperature of the sample. By reducing the temperature of the sample, the ambient RH in the vicinity of the sample increases, and therefore, more water molecules adsorb onto the surface. Working at $0 \%$ RH minimizes any possible increase in the amount of water adsorbed when changing the temperature of the sample and consequently, keeping the diffusion of the ionic species, and the screening speed, independent of the sample temperature.

When we reduced the sample temperature, the CPD contrast between the polarized and non-polarized areas changed dramatically. In the example illustrated in Fig. 2, after surface screening at ambient conditions, the chamber was filled with 


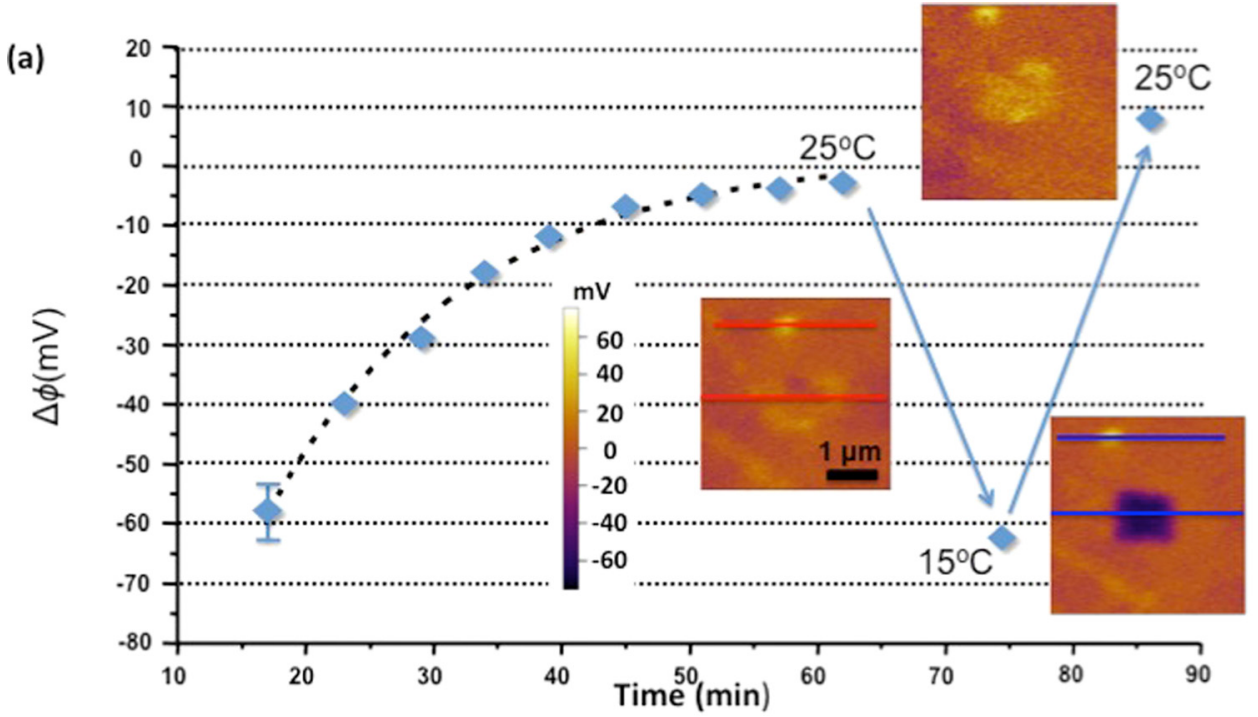

(b)

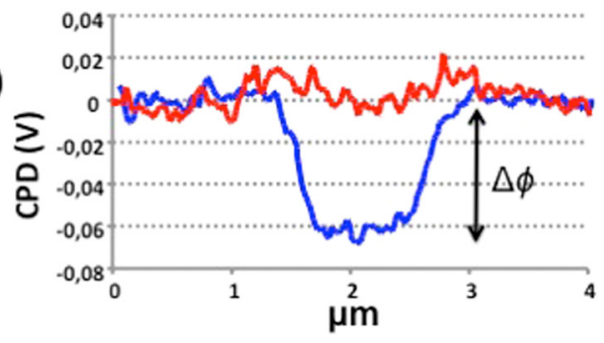

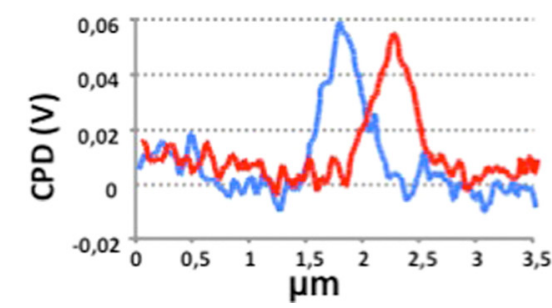

FIG. 2. (a) Change in the surfacepotential contrast $(\Delta \phi)$ of the polarized and the non-polarized regions over time. Surface charges diffuse to screen the polarization charge following a slow decay (timescale of tens of minutes). The dashed line indicates exponential fitting of the decay. Once the equilibrium had been reached, the temperature was dropped from $25^{\circ} \mathrm{C}$ to $15^{\circ} \mathrm{C}$, and a new, negative contrast was measured. (b) Profiles of the contrast in the polarized region and on the contamination spot. As the temperature decreased, the contrast in the polarized region changed while that in the spot remained constant. dry $\mathrm{N}_{2}$ and an almost null contrast value $(\Delta \phi \sim-3 \mathrm{mV})$ was reached, then the temperature was subsequently decreased from $25^{\circ} \mathrm{C}$ to $15^{\circ} \mathrm{C}$. The KPFM image taken immediately after the temperature had been decreased revealed a negative CPD contrast $(\Delta \phi=-63 \mathrm{mV})$. Contrariwise, the contrast in the contamination spot, which was used as a reference [see profiles in Fig. 2(b)], remained unchanged, indicating that the observed variations in CPD correspond to real changes in the polarized region of the sample and not to artifacts from the measurements. When the temperature was raised back up to $25^{\circ} \mathrm{C}$, a subsequently captured KPFM image indicated a slightly positive CPD contrast $(\Delta \phi=10 \mathrm{mV})$.

Figure 3 illustrates a detailed study of the CPD contrast between the polarized and non-polarized regions as a function of temperature. As in our previous experiments, we performed the polarization under ambient conditions $\left(25^{\circ} \mathrm{C}\right.$ and $40 \% \mathrm{RH})$. Dry $\mathrm{N}_{2}$ was then introduced into the chamber, and the temperature was reduced to $10^{\circ} \mathrm{C}$. The stable potential contrast was established by taking consecutive KPFM images under these conditions, until two consecutive images showed the same surface-potential contrast (with an error of $\pm 5 \mathrm{mV}$ in the contrast, and with a difference of $6 \mathrm{~min}$ between images). Then the temperature dependence of the contrast was studied by taking an image of a KPFM contrast of the polarized region by scanning the same line at $0.75 \mathrm{~Hz}$ (slow scan disabled) as a function of the temperature [Fig. $3(\mathrm{a})$. The temperature was increased in a monotonous ramp of $2.5^{\circ} \mathrm{C}$ per minute starting at $10^{\circ} \mathrm{C}$ (bottom of the image) and finishing at $25^{\circ} \mathrm{C}$ (top of the image). Immediately after the first image had been taken, a second image was taken in which the temperature ramp was reversed from $25^{\circ} \mathrm{C}$ (top of the image) down to $10^{\circ} \mathrm{C}$ (bottom of the image). As observed in the figure, the contrast changed from negative to positive as the temperature increased, and back to negative as the temperature was decreased again. The curvature of the polarized region observed in the images as the temperature changed is due to thermal drift of the system. The bottom of Fig. 3(a) shows the change in the surface-potential contrast between the polarized and non-polarized regions extracted from the images. As observed, the change is nonlinear with temperature and is characterized by hysteresis: the contrast after the temperature ramps up and down does not return to the original value of $\Delta \phi=-70 \mathrm{mV}$ at $10^{\circ} \mathrm{C}$ and shows a larger, negative contrast of $\Delta \phi=-120 \mathrm{mV}$. Another image taken immediately after finishing the cooling ramp-down to $10^{\circ} \mathrm{C}$, during which time the temperature was held constant [Fig. 3(b)], shows that the contrast decreases with time, ultimately stabilizing at $\Delta \phi=-65 \mathrm{mV}$, approximately equal to the previous original value. Note that only the CPD value on the polarized region changes with time. When the sample temperature was held constant at $25^{\circ} \mathrm{C}$ and the humidity was increased to $\sim 80 \%$, a general screening of the potential on the contamination spot and the polarized region was observed as a strong reduction in contrast in the KPFM image (Fig. 4). When the humidity was lowered again, the contrast returned to its previous level. Similar results have been reported in AFM studies of water adsorption onto the surfaces of $\mathrm{BaTiO}_{3}(001)$ single crystals. ${ }^{20}$

We tracked the change in surface-potential contrast with temperature and with time by repeating temperature cycles in a single experiment (see Fig. 5). We discuss the results in detail below, but we would first like to underscore three important trends: (i) the contrast gradually shifts to more positive values; (ii) the difference in contrast at the higher and lower temperatures decreases with each successive 
(a)
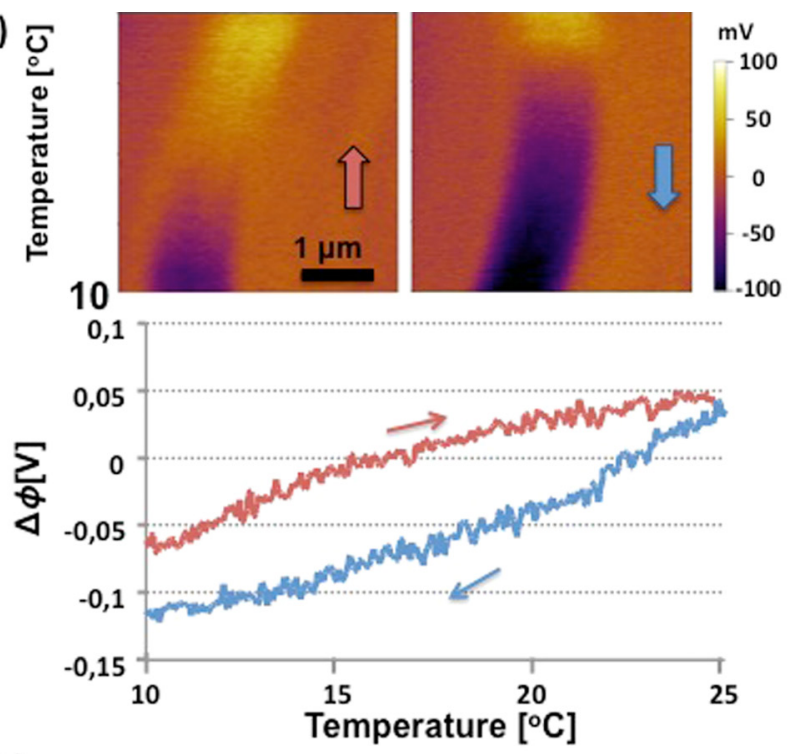

(b)
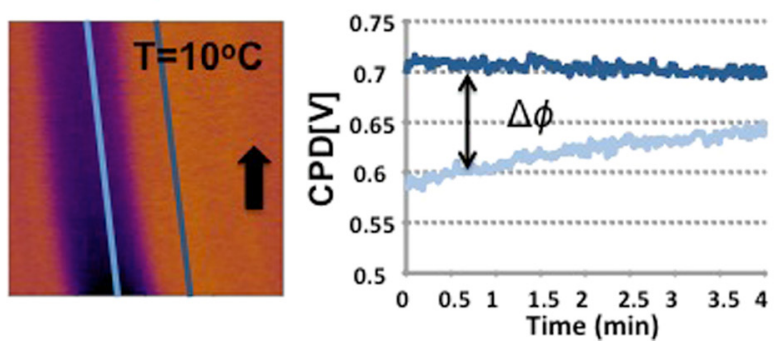

FIG. 3. (a) (Top) KPFM images showing consecutive surface potential profiles of a polarized region as the temperature was increased from $10^{\circ} \mathrm{C}$ to $25^{\circ} \mathrm{C}$ (left) and subsequently decreased back to $10^{\circ} \mathrm{C}$ (right). (Bottom) a plot of the contrast in the surface potential between the polarized and nonpolarized regions $(\Delta \phi)$ shows a difference in the value after temperature had been increased and subsequently decreased. (b) (left) Image showing consecutive surface potential profiles over time at $10^{\circ} \mathrm{C}$, acquired immediately after acquisition of the images in (a). (Right) Profiles of the image versus time showing how contact potential value in the non-polarized region remained constant while that in the polarized region increased due to screening. The final contrast value $(\Delta \phi)$ is very similar to the original value, which obtained at the same temperature at the beginning of the left image in (a).

temperature cycle; and (iii) the curves of contrast plotted as a function of temperature gradually change shape.

\section{DISCUSSION}

Our results show that when a change in polarization is induced using an AFM tip, screening of the polarizationbound charge can be rather long (on the order of tens of minutes under ambient conditions). This indicates that fast screening processes such as band bending, or reorientation of water molecule dipoles, cannot completely screen the dipole and that a surface diffusion of charges is needed to reach an equilibrium state. Changing the temperature of the sample disrupts the equilibrium, because polarization-bound charge is temperature dependent: the lower the temperature, the larger the dipole. We found that the change in polarizationbound charge leaves some of the screening charge uncompensated, as has already been reported for other ferroelectric materials, such as $\mathrm{BaTiO}_{3}{ }^{3}$ Following the charge dynamics model proposed for $\mathrm{BaTiO}_{3}$, we can explain the results shown in Figures 2 and 3 as follows. In Figure 2, an equilibrium value had been reached at $25^{\circ} \mathrm{C}$, and then the temperature was decreased to $15^{\circ} \mathrm{C}$. At this point, the contrast became more negative, because the surface charges that had been compensating the polarization-bound charge at $25^{\circ} \mathrm{C}$ were not able to immediately compensate the increase in polarization; as such, the corresponding KPFM images show a strong negative contrast. When temperature was raised back to $25^{\circ} \mathrm{C}$, a positive contrast was observed that had not been observed in the first image taken at $25^{\circ} \mathrm{C}$. During acquisition of the image at $15^{\circ} \mathrm{C}$, some of the surface charges already diffused to compensate the increase in the polarization-bound charge; thus, when the temperature was raised again, there was an excess of positive charges (i.e., the polarization-bound charge was overscreened), thereby producing a positive contrast in the image (see the screening charge dynamics schemes in Fig. 5). This finding is corroborated by the results shown in Fig. 3, in which hysteresis in the plots of contrast versus temperature cycle is observed, and the original value was recovered when the system had been left to relax.

Differences in surface-potential contrast $(\Delta \phi)$ of $\sim 100 \mathrm{mV}$ have been correlated to a screening dipole layer consistent with water layer having a thickness of a few molecules. ${ }^{9}$ It has been predicted that such a water layer should exist on many surfaces at ambient conditions. ${ }^{2}$ However, screening due to water dipoles is generally expected to occur almost instantaneously when compared with our acquisition time, and it would have a limited screening capability. Diffusion of ionic species inside this water layer can be an important source of screening charges that would have a response time of a few minutes, as has already been reported for other surfaces. Once adsorbed onto the surface, the first water monolayers can remain there, even under low humidity conditions, although diffusion in such thin water layers is generally expected to be more limited. If a thick film of water is present on a surface under high $\mathrm{RH}$, then water can screen all the charges and homogenize the surface-potential contrast in the entire image (Fig. 4). However, one must take into account that the charge-screening capability of the water
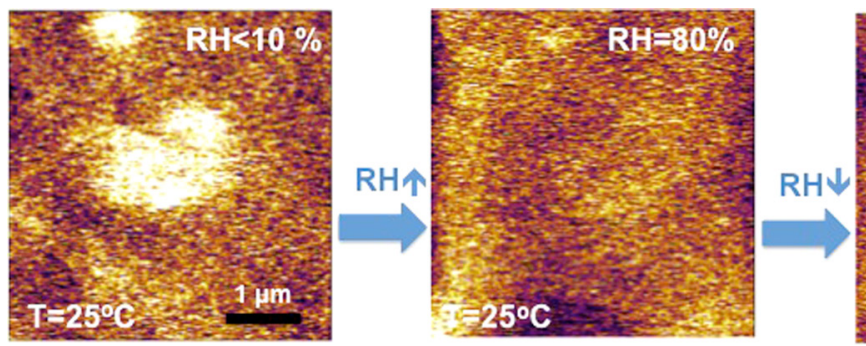

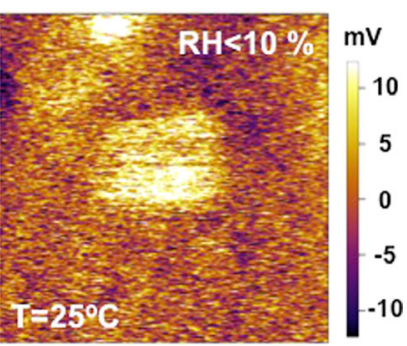

FIG. 4. KPFM images of the polarized region in Figs. 1 and 2 acquired at $25^{\circ} \mathrm{C}$, showing bright contrast due to screening surface charge. When the humidity was increased up to $70 \%$, the adsorbed water screened all the contrast, including the contamination spot. When the humidity was subsequently reduced to low levels, the previous contrast value was reestablished. 


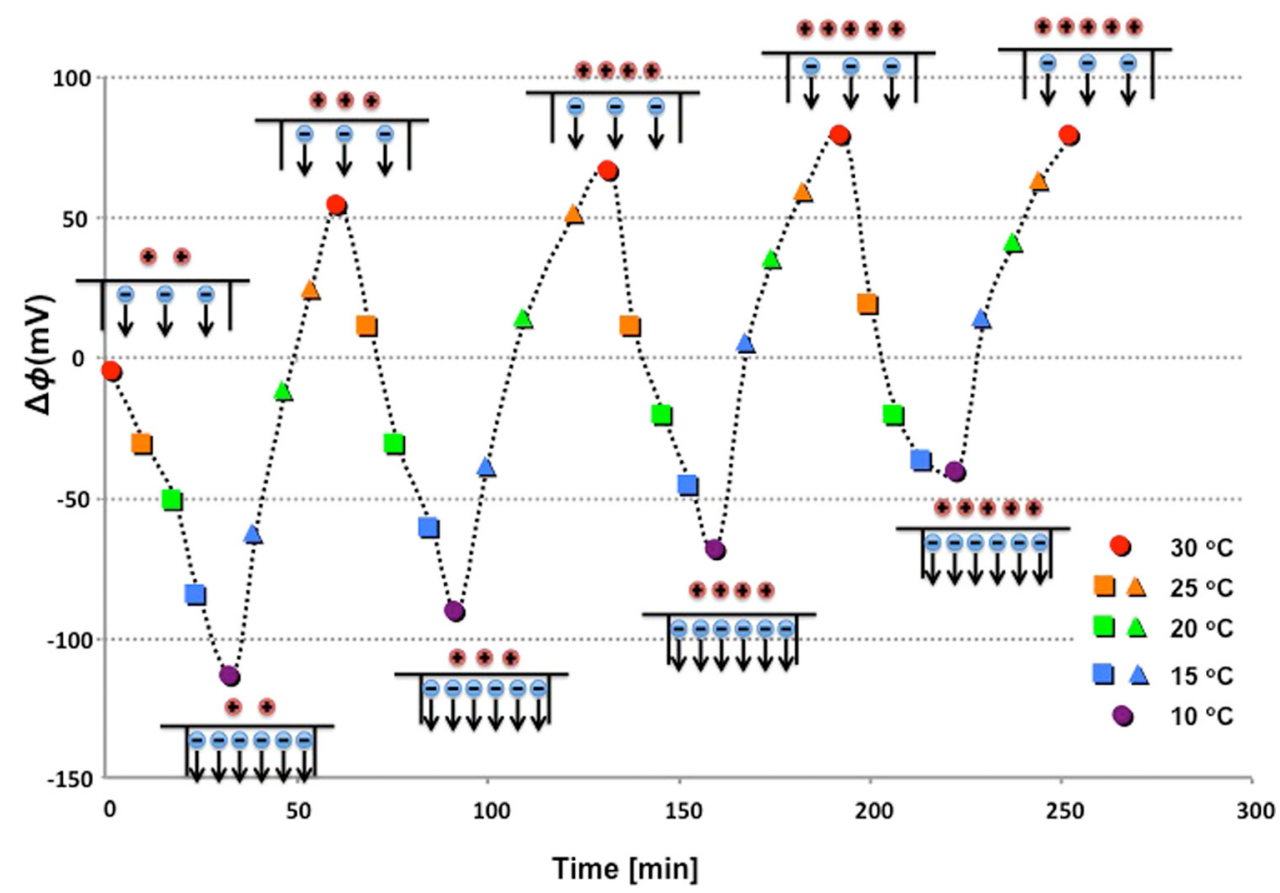

FIG. 5. Change in the surface-potential contrasts between a polarized and a nonpolarized region ( $\Delta \phi$ in Fig. 2) over time. Immediately, after polarization at $30^{\circ} \mathrm{C}$, successive temperature ramps were performed: first down to $10^{\circ} \mathrm{C}$, and then back up to $30^{\circ} \mathrm{C}$. Images were acquired at selected temperatures: $30^{\circ} \mathrm{C}$, $25^{\circ} \mathrm{C}, 20^{\circ} \mathrm{C}, 15^{\circ} \mathrm{C}$, and $10^{\circ} \mathrm{C}$. The illustration shows the changes in the polarization of the sample and in the screening charge dynamics as functions of temperature and time.

molecules dipole depends on the interactions between these water molecules and the surface. Thin films of water on some surfaces can be forced to adopt highly oriented structures $^{21}$ that can induce-even at room temperatures-the formation of ferroelectric layers that would enhance the surface dipole. $^{22}$ It has been reported that the presence of surface dipoles on amino acid crystals determines the interaction of water with the surface ${ }^{23}$ and that surface dipoles on pyroelectric materials $^{24}$ and polar amino acid crystals ${ }^{25}$ catalyze the freezing of water at higher temperatures. In our experiments, we kept the temperature above $0{ }^{\circ} \mathrm{C}$ to avoid such effects; nevertheless, on certain surfaces some degree of water structuration due to the substrate can happen well above the freezing point. ${ }^{26}$

The nature of the ionic species on the surface is still under debate. ${ }^{4,27}$ Surface dissociation of water is generally expected to occur during polarization, whereby a water neck is formed between the tip and the sample, and a voltage of a few volts is applied between the sample and the tip. Additionally, exposure to water vapor can induce changes in the chemistry and the structure of the film surface. Dissociative adsorption of water has been already proven to be an effective screening mechanism on single crystals of $\mathrm{BaTiO}_{3}(001){ }^{28} \mathrm{BaTiO}_{3}$ films exposed to water were found to induce surface hydroxylation and formation of oxygen vacancies that lead to formation of a dead, non-ferroelectric surface layer. $^{27}$

We observed that at all temperatures, the surfacepotential contrast gradually shifted to more positive values (see data in Fig. 5). This trend apparently reached equilibrium in the final cycles of the experiment, especially for higher temperatures: for example, in the last two data points, the contrast values at $30^{\circ} \mathrm{C}$ are almost equal (data taken at 190 and $250 \mathrm{~min}$ ). These shifts suggest that positive charges incorporate into the surface with time. Since we performed our experiments in a controlled, non-reactive environment (dry $\mathrm{N}_{2}$ ), we consider it unlikely that such charges were new adsorbates from the environment. In fact, we believe that these charges are most likely created on the surface, either at the PZT interface or from long range diffusion from other regions of the sample. During polarization, some positive charges were incorporated to the sample. Such charges are not localized to the polarized region, but rather can diffuse during polarization to the area surrounding the $1 \mu \mathrm{m} \times 1 \mu \mathrm{m}$ polarized square. This effect can be observed in the image in Fig. 2(a) at $25^{\circ} \mathrm{C}$, which reveals a weak negative contrast on the polarized square, which is surrounded by a positive contrast due to charge injection. At low humidity, surface diffusion of charges can be very slow and therefore, these charges may take some time to reach the polarized region.

The slow diffusion of surface charges is evident in the decay rate of the potential versus temperature cycles in Fig. 5. After each temperature ramp inversion, the surfacepotential contrast behavior resembles that of the charge/discharge process of a capacitor, characterized by strong initial variation. Since temperature-dependent variation in the bond polarization charge can be considered to be instantaneousand thus, linear-in this temperatures range, ${ }^{8}$ the relaxation must come from the dynamics of charge screening. One possible explanation for this relaxation is the acceleration and deceleration undergone by the adsorbate charges: apparently, these charges can screen variations in the polarization-bound charge faster if they are already moving towards or away from the polarized region. Moreover, screening processes that require inversion of the charge diffusion direction are slower.

The externally tip-induced polarization in our experiments is stable over our measurement timescale. However, lowering the temperature induces unbalanced surface charges that create large electric fields that could be strong enough to overcome the energy barrier to locally switch polarization in order to relax the system. Although we did not observe any 
change in the polarization within the lateral resolution of the experiments, we cannot rule out the possibility that small nanodomains of opposite polarization had formed within the polarized region to relax the system. In fact, such a scenario could explain why the difference between the values for surface-potential contrast at $30^{\circ} \mathrm{C}$ and $10^{\circ} \mathrm{C}$ decreases with each temperature cycle (see Fig. 5).

\section{CONCLUSIONS}

We have studied screening of polarized domains on ferroelectric PZT thin films using PFM and KPFM. New domains can be created using AFM lithography showing minimum charge injection. The sign of the surface potential contrast of the domains created under these conditions corresponds to the expected sign of the created dipoles and it becomes gradually screened with time by surface charges until null or opposite contrast is measured. Relaxation time of this process under ambient conditions was found to be on the order of minutes, consistently with discharging processes associated to the diffusion of ionic species in the water layer adsorbed onto the surface. When the sample was subject to temperature cycles, variations in the surface-potential measurements were observed; there were attributed to temporally unbalanced surface screening charges. These results are consistent with those of previously published experiments on $\mathrm{BaTiO}_{3}$ (100). Measurements taken under high humidity indicated that thick water layers can completely screen surface charges, thus making surface-potential contrast homogeneous. Finally, the dynamics of charge screening was studied in a dry $\mathrm{N}_{2}$ environment. Slower dynamics were found when the temperature variation was reversed in a temperature cycle. It was found that positive charges incorporated into the surface during temperature cycles, which was attributed to the creation or difussion of ionic species from the sample itself, as the environment was non-reactive. This work calls for future studies on the nature of adsorbates on polarized PZT thin films under controlled environmental conditions using KPFM and other surface-sensitive techniques. Studies on screening under different gas environments and at different temperatures should help elucidate the nature and dynamics of the surface adsorbates in ferroelectrics.

\section{ACKNOWLEDGMENTS}

We thank Professor G. Catalan for useful discussions and acknowledge Professor S. Kalinin's guidance in helping us to understand the charge screening processes in ferroelectrics. This work was supported by the former Spanish Goverment through Project No. FIS2009-08355 and Project
No. MAT2010-17771. N.D. acknowledges financial support from an RyC Grant of the Spanish Ministerio de Economía y Competitividad (MINECO).

${ }^{1} \mathrm{~J}$. N. Israelachvili, Intermolecular and Surface Forces: With Applications to Colloidal and Biological Systems (Academic Press, London, 1985).

${ }^{2}$ A. Verdaguer, G. M. Sacha, H. Bluhm, and M. Salmeron, Chem. Rev. 106(4), 1478-1510 (2006).

${ }^{3}$ S. V. Kalinin and D. A. Bonnell, Nano Lett. 4(4), 555-560 (2004).

${ }^{4}$ M. J. Highland, T. T. Fister, D. D. Fong, P. H. Fuoss, C. Thompson, J. A. Eastman, S. K. Streiffer, and G. B. Stephenson, Phys. Rev. Lett. 107(18), 187602 (2011).

${ }^{5}$ G. B. Stephenson and M. J. Highland, Phys. Rev. B 84(6), 064107 (2011).

${ }^{6}$ E. A. Eliseev, A. N. Morozovska, G. S. Svechnikov, E. L. Rumyantsev, E. I. Shishkin, V. Y. Shur, and S. V. Kalinin, Phys. Rev. B 78(24), 245209 (2008).

${ }^{7}$ S. V. Kalinin and D. A. Bonnell, J. Appl. Phys. 87(8), 3950-3957 (2000).

${ }^{8}$ S. V. Kalinin and D. A. Bonnell, Appl. Phys. Lett. 78(8), 1116-1118 (2001).

${ }^{9}$ S. V. Kalinin and D. A. Bonnell, Phys. Rev. B 63(12), 125411 (2001).

${ }^{10}$ D. Y. He, L. J. Qiao, and A. A. Volinsky, J. Appl. Phys. 110(7), 074104 (2011).

${ }^{11}$ D. Y. He, L. J. Qiao, A. A. Volinsky, Y. Bai, and L. Q. Guo, Phys. Rev. B 84(2), 024101 (2011).

${ }^{12}$ T. Tybell, C. H. Ahn, and J. M. Triscone, Appl. Phys. Lett. 75(6), 856-858 (1999).

${ }^{13}$ E. Soergel, J. Phys. D: Appl. Phys. 44(46), 464003 (2011).

${ }^{14}$ C. H. Kim, K. S. Song, H. B. Moon, J. Y. Son, B. G. Kim, and J. H. Cho, J. Korean Phys. Soc. 51(2), 687-691 (2007).

${ }^{15}$ J. Y. Son, S. Min, C. H. Kim, B. G. Kim, and J. H. Cho, J. Korean Phys. Soc. 51(2), 655-658 (2007).

${ }^{16}$ A. Verdaguer, J. J. Segura, J. Fraxedas, H. Bluhm, and M. Salmeron, J. Phys. Chem. C 112(43), 16898-16901 (2008).

${ }^{17}$ B. J. Rodriguez, C. Callahan, S. V. Kalinin, and R. Proksch, Nanotechnology 18(47), 475504 (2007).

${ }^{18}$ S. Santos, A. Verdaguer, T. Souier, H. N. Thomson, and M. Chiesa, Nanotechnology 22(46), 465705-465713 (2011).

${ }^{19}$ A. Verdaguer, M. Cardellach, J. J. Segura, G. M. Sacha, J. Moser, M. Zdrojek, A. Bachtold, and J. Fraxedas, Appl. Phys. Lett. 94(23), 233105 (2009).

${ }^{20}$ D. Y. He, L. J. Qiao, A. A. Volinsky, Y. Bai, M. Wu, and W. Y. Chu, Appl. Phys. Lett. 98(6), 062905 (2011).

${ }^{21}$ A. Verdaguer, C. Weis, G. Oncins, G. Ketteler, H. Bluhm, and M. Salmeron, Langmuir 23(19), 9699-9703 (2007).

${ }^{22}$ H. Bluhm, T. Inoue, and M. Salmeron, Surf. Sci. 462(1-3), L599-L602 (2000).

${ }^{23}$ J. J. Segura, A. Verdaguer, M. Cobián, E. R. Hernández, and J. Fraxedas, J. Am. Chem. Soc. 131(49), 17853-17859 (2009).

${ }^{24}$ D. Ehre, E. Lavert, M. Lahav, and I. Lubomirsky, Science 327(5966), 672-675 (2010).

${ }^{25}$ R. Popovitzbiro, J. L. Wang, J. Majewski, E. Shavit, L. Leiserowitz, and M. Lahav, J. Am. Chem. Soc. 116(4), 1179-1191 (1994).

${ }^{26}$ A. Verdaguer, M. Cardellach, and J. Fraxedas, J. Chem. Phys. 129(17), 174705 (2008).

${ }^{27}$ J. Shin, V. B. Nascimento, G. Geneste, J. Rundgren, E. W. Plummer, B. Dkhil, S. V. Kalinin, and A. P. Baddorf, Nano Lett. 9(11), 3720-3725 (2009).

${ }^{28}$ J. L. Wang, A. Pancotti, P. Jegou, G. Niu, B. Gautier, Y. Y. Mi, L. Tortech, S. Yin, B. Vilquin, and N. Barrett, Phys. Rev. B 84(20), 205426 (2011). 